\title{
Percutaneous Endoscopic Transforaminal Lumbar Discectomy - An Early Experience
}

\author{
CC Wong, MS (Ortho), WP Loke, MS (Ortho) \\ Department of Orthopaedics, Sarawak General Hospital, Kuching, Sarawak, Malaysia
}

\begin{abstract}
Percutaneous endoscopic spinal surgery performed in the awake state offers a new paradigm for treatment of symptomatic lumbar disc prolapse. We report the outcome of 23 patients who underwent this procedure. Visual analogue scale for pain improved from 7.3 to $2.1 ; 19$ of the 23 patients achieved good to excellent results according to the MacNab criteria. Patient acceptance of the procedure was $91.3 \%$. All but one patient were discharged from hospital within 24 hours. One patient developed foot drop post-operatively. There was no incidence of dural tear, post-operative infection or worsening of symptoms. We conclude that this is a safe, effective, and well-tolerated procedure.
\end{abstract}

\section{Key Words:}

Percutaneous endoscopic lumbar discectomy, Lumbar disc prolapse, Transforaminal approach, Minimally invasive spine surgery

\section{INTRODUCTION}

Lumbar disc prolapse with radicular pain or sciatica is a common clinical problem. Although the majority of patients improve with conservative (non-surgical) therapy, many patients continue to experience functionally limiting pain. Open surgical procedures are associated with operative risk, such as nerve root injury, vascular complications and epidural fibrosis ${ }^{1}$, and it takes time for patients to recover from surgical trauma to the paraspinal structures. It therefore has become more common for spine surgeon to consider minimally invasive procedures for these patients ${ }^{2}$. Percutaneous endoscopic surgery has several advantages over open surgery, including clear visualization and targeted fragmentectomy ${ }^{3}$. There is also less damage to the paraspinal muscles with this technique and the procedure can be performed utilizing awake state anaesthesia with on-going patient feedback. Thus, there is reduced risk of major nerve root injury. Patients were able to return to work earlier after the minimally invasive procedure as compared to more traditional methods ${ }^{4}$. The aim of the present study was to investigate early experience using of percutaneous endoscopic lumbar discectomy for the surgical treatment of symptomatic lumbar disc prolapse in Sarawak General Hospital in Kuching, Sarawak, Malaysia.

\section{MATERIALS AND METHODS}

This is a prospective outcome assessment of 23 consecutive cases of percutaneous endoscopic lumbar discectomy performed in Sarawak General Hospital since the necessary equipment became available. We performed percutaneous endoscopic lumbar discectomy using a transforaminal approach in 23 patients who fulfilled the inclusion criteria. These procedures took place between October 2005 and June 2006.

\section{Inclusion Criteria}

Patients included in this study met the following criteria: 1) Unilateral radiating leg pain that was more severe than axial back pain; 2) Failure of conservative management to relieve pain; 3) Magnetic resonance imaging (MRI) scan of the lumbar spine confirming posterolateral disc prolapse correlating to clinical presentation.

\section{Exclusion criteria}

Patients were excluded from this study based on the following criteria: 1) Evidence of central or lateral canal spinal stenosis on CT or MRI scans; 2) Multiple level disc protrusion; 3) Significant motor deficit; 4) Radiological evidence of segmental instability; 5) Previous open surgery at the same level; or 6) Infection, tumour or fracture associated with the disc prolapse.

\section{Surgical technique}

All procedures were performed under local anaesthesia. Patient was sedated with either pethidine with midazolam or with awake state anaesthesia utilizing propofol infusion. Patient was positioned prone on a radiolucent table. The image intensifier was set into Fergusson view (caudal tilt of 15 to 25 degrees) so that the desired level endplates were seen tangentially. The midline was marked along the tips of transverse processes while ensuring that the left and right pedicles were equidistant from the midline in Fergusson view of the spine. 
Skin puncture was made approximately $11 \mathrm{~cm}$ from the midline. Using lateral view fluoroscopic guidance, the spinal needle was advanced towards the intervertebral disc, avoiding both the traversing and the exiting nerve roots. Confirmation of intradisc placement of the needle tip was achieved by discography (using a mixture of indigo carmine dye and radiopaque contrast [Omnipaque]). The indigo carmine dye stained the degenerated disc material, thus aiding in the procedure.

A guide wire was then inserted through the spinal needle after removal of the stylet. A $7 \mathrm{~mm}$ skin incision was made to enable the passage of the spine scope sheath. The $7.2 \mathrm{~mm}$ fenestrated working cannula was inserted after serial dilatation. Fluoroscopic guided discectomy was performed using a $6 \mathrm{~mm}$ diameter grasper in the $7.0 \mathrm{~mm}, 20$ degrees viewing angle of the spine endoscope (Arthrokinetic, Germany). Constant gravity flow saline irrigation through the sheath and scope helped to reduce bleeding induced clouding of the operating field.

Herniated disc material was removed using $3.5 \mathrm{~mm}$ diameter forceps and graspers inserted through the $4.0 \mathrm{~mm}$ working channel of the endoscope, under direct endoscopic visualization. Patient communication was maintained throughout the procedure to ensure nerve root safety and relief of radicular pain. Either a radiofrequency bipolar probe (Arthrokinetic, Germany) or a side firing HolmiumYAG laser probe (Coherent Inc, USA) were used to shrink soft tissue to improve operating field visualization. The end point of the procedure was visualization of spontaneous dural pulsation, dural cough impulse or relief of radicular pain as reported by the patient.

\section{Outcome analysis}

Patients were evaluated using the visual analogue scale (VAS) for pain and the modified MacNab score for outcome measure (Need citation for MacNab score). They were assessed pre-operatively, immediate post-operatively, at one month and three months after the procedure. Patient acceptance of the procedure was assessed by asking the patients if they would be willing to undergo the same procedure again.

\section{RESULTS}

There were 10 male and 13 female patients in the present study. The average age was 44 years, range $28-53 y$. Thirteen patients had prolapsed disc at L4-5 level, whereas 10 patients had L5-S1 disc prolapse. Mean pre-operative VAS was 7.3 (range 6-10), and the immediate post-operative VAS was 2.1 (range 0-4). VAS at one month was 2.4 (range 0-4).

The modified MacNab score at one month post-operation was excellent in 5, good in 14, fair in 4 with none in the poor group. Considering the number of subjects with excellent or good scores as favourable outcome, 19 out of the 23 patients $(82.6 \%)$ in the present study had a favourable outcome. All except one patient were discharged from the hospital within 24 hours.

It was not necessary to abandon surgery before completion for any of the study subjects. Two patients complained of severe pain during the procedure but because of the amnesic effect of the sedatives, both of them did not recall the uncomfortable experience. One patient developed foot drop post-operatively. The neurological deficit was transient, with complete recovery of the deficit noted at 6 weeks after the operation. Four patients reported numbness of the leg despite resolution of sciatica pain. One patient had early recurrent disc prolapse after doing well for 3 months postoperatively. There was no incidence of infection in this series of surgeries, nor were there any cases of vascular complication or dural leak. No patient reported worsening of symptoms.

The mean duration of follow up was 17 weeks. By the time of the last follow up visit, there was no case of infection or neural deficit. One patient developed radicular pain on the contralateral side at the surgical level two months after surgery, despite reporting good symptomatic improvement on the operative side. He subsequently underwent open posterior decompression with good outcome.

21 out of 23 patients $(91.3 \%)$ regarded this procedure as tolerable and were willing to undergo the same procedure again should the need arise.

\section{DISCUSSION}

Open lumbar microdiscectomy is the gold standard for treatment of symptomatic lumbar disc prolapse and has a reported success rate of eighty to ninety-six percent ${ }^{5,6}$. Percutaneous transforaminal endoscopic techniques performing targeted fragmentectomy under local anaesthesia using fluoroscopic guided endoscopy have evolved over the past three decades ${ }^{7.8}$. Over this period of time, improved endoscope optics and new miniaturized instruments have enabled the technique to be introduced into the clinical setting. The surgical goal for both procedures is the same, namely to decompress the nerve root by removing the offending prolapsed disc fragments. Despite the fact that the present study is initial series taking into account the surgeon's learning curve, the success rate was $82.6 \%$. This is comparable to the result of the gold standard open microdiscectomy.

With the patient in an awake state of anaesthesia, continuous verbal communication with the operating surgeon was possible. This increases the safety of the procedure, as the nerve roots are not anaesthetized by the $0.5 \%$ local anaesthetic used. Patient may experience pain, or increased 
Table I: Modified MacNab criteria for characterizing outcome after spinal surgery

\begin{tabular}{|ll|}
\hline Grade & Description of criteria \\
\hline Excellent & Free of pain, no mobility restriction, able to return to work \\
Good & Occasional non-radicular pain, relief of presenting symptoms, able to return to modified work \\
Fair & Some improved functional capacity, still handicapped or unemployed \\
Poor & Continued objective symptoms of root involvement, additional operative intervention needed at index level \\
\hline
\end{tabular}

Table II: Demographics of cases

\begin{tabular}{|lcc|}
\hline Level & Males & Females \\
\hline L4-5 & 6 & 7 \\
L5-S1 & 4 & 6 \\
Subtotal & 10 & 13 \\
\hline
\end{tabular}

Table III: Complications of percutaneous endoscopic lumbar discectomy (PELD)

\begin{tabular}{|l|c|}
\hline Complications & No \\
\hline Transient motor deficit & 1 \\
Transient numbness & 4 \\
Contralateral radicular pain & 1 \\
Recurrent disc prolapse & 1 \\
\hline
\end{tabular}

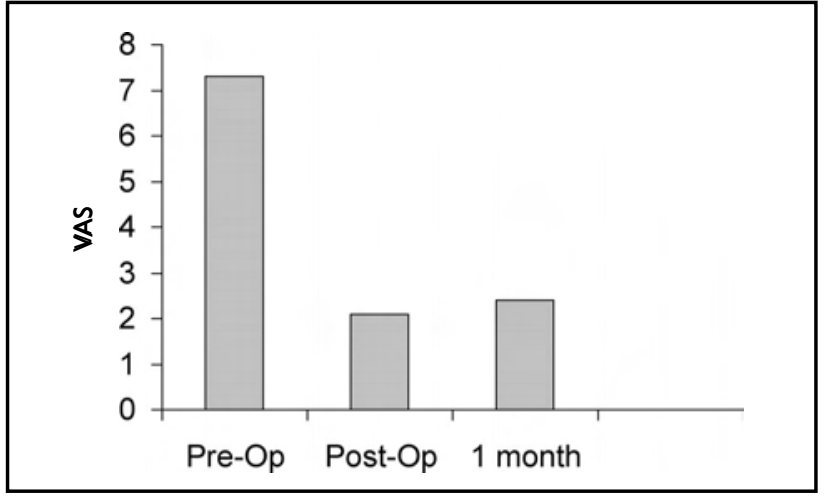

Fig. 1: Pre-operative and post-operative visual analogue scale.

numbness or heaviness of the leg when the nerve root is threatened. In the present study, there was one patient with post-operative L5 weakness, confirmed by electrophysiological study whose procedure was performed at L5-S1 level. The exiting L5 nerve root was injured by the working cannula. This patient developed hypoxia from over sedation with pethidine and midazolam, and attempted to get up from the prone position with the endoscope still in the intervertebral disc space. After correcting the hypoxia, the procedure was continued but no communication was possible with the patient due to over sedation. The patient subsequently underwent open foraminotomy at the L5-S1 level to explore the nerve roots because of the neural deficit; it was found that the nerves were not severed. The weakness was completely resolved by week 6 postoperatively. In our opinion, this is a preventable event with close monitoring of the patient.

Four patients complained of numbness of the leg after the procedure despite good resolution of leg pain. At the latest

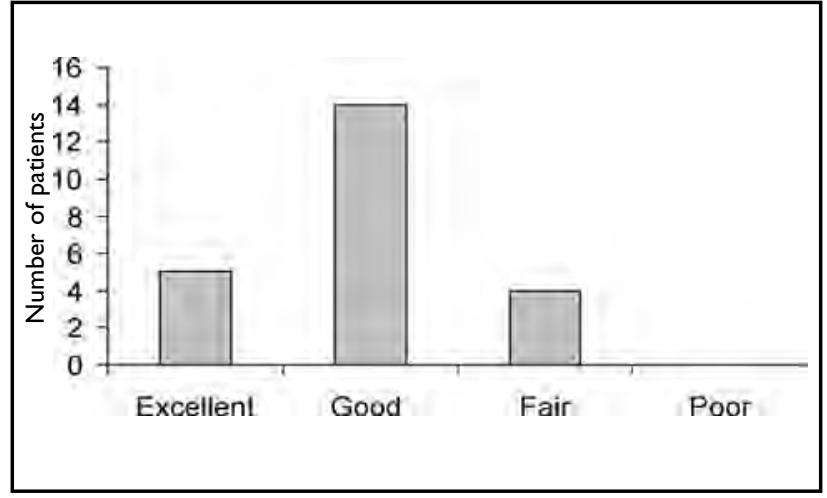

Fig. 2: Modified MacNab score at one month post-operation.

follow up, 3 patients reported significant improvement of the numbness. We postulate that these patients only noticed the numbness after the severe radicular pain was relieved by the procedure. However, we caution that this may possibly be due to exiting nerve root injury by the working cannula, ${ }^{9,10}$. One patient returned to the clinic reporting increasing leg pain at 3 months post-operatively following initial resolution of symptom. Repeat MRI scan with contrast showed a recurrent disc prolapse. He was offered but declined to undergo an open procedure for the recurrent disc prolapse.

We are not able to explain why one patient developed contralateral radicular pain despite satisfactory resolution of symptom after the percutaneous procedure. This 51 year old patient underwent L4-5 percutaneous endoscopic discectomy for right L5 radicular pain. He complained of left L5 radicular pain at 2 months after surgery. Repeat MRI scan did not show new disc prolapse, but did reveal mild ligamentum flavum thickening. Dynamic lateral lumbar radiographs also did not demonstrate segmental instability. 


\section{CONCLUSION}

Percutaneous endoscopic transforaminal lumbar discectomy is an effective and safe minimally invasive procedure for the treatment of symptomatic lumbar disc prolapse. Careful selection of the patients is essential to ensure favourable outcome with minimal morbidity. Maintaining an awake state of anaesthesia is a vital component of the procedure as this allows the patient to report any threat to the nerve roots.

\section{REFERENCES}

1. Saal JA, Saal JS. Nonoperative treatment of herniated lumbar interevertebral disc with radiculopathy: An outcome study. Spine 1989; 14: 431-7.

2. Maroon JC. Current concept in minimally invasive discectomy. Neurosurgery 2002; 51: 137-45.

3. Yeung AT, Tsou PM. Posterolateral endoscopic excision for lumbar disc herniation: Surgical technique, outcome, and complications in 307 consecutive cases. Spine 2000; 27: 722-31.

4. Chiu JC, Clifford TJ, Betterjee KA, Princenthal RA. Extradural transpinal percutaneous L5-S1 endoscopic discectomy, in Savitz M H, Chiu J C, Yeung A T (eds). The Practice of Minimally Invasive Spine Surgery. American Academy of Minimally Invasive Spinal Medicine and Surgery, 3ima, CSS, 2000, 227-30.

5. McCulloch JA. Focus issue on lumbar disc herniation: Macro- and microdiscectomy. Spine 1996; 21: S45-S56.

6. Gibson JN, Waddell G. Surgical interventions for lumbar disc prolpase. Cochrane Database Syst Rev. 2007 Jan 24; (1): CD001350.

7. Schreiber A, Suezawa Y, Leu H. Does percutaneous nucleotomy with discoscopy replace conventional discectomy? Eight years of experience and results in treatment of herniated lumbar disc. Clin Orthop 1989; 238: 35-42.

8. Kambin P, Brager MD. Percutaneous posterolateral discectomy. Anatomy and mechanism. Clin Orthop 1987; 223: 145-54.

9. Choi G, Lee SH, Raitruker. Percutaneous endoscopic interlaminar discectomy for intracanalicular disc herniations at L5-S1 using a rigid working channel endoscope. Neurosurg 2006; 58(1): 59-68.

10. Min JH, Kang SH, et al. Morphometric analysis of the working zone for endoscopic lumbar discectomy. J Spinal Disord 2005; 18(2): 132-5. 